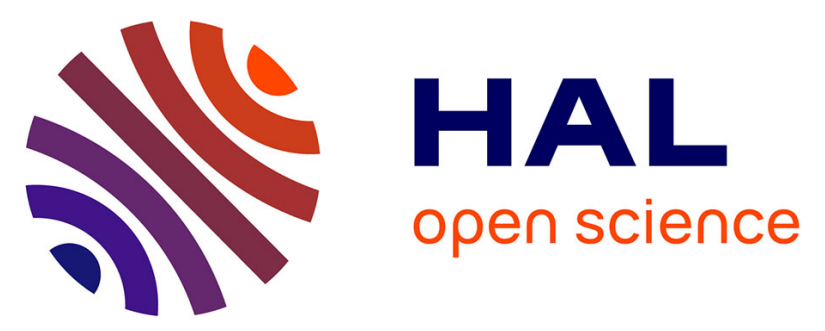

\title{
Hyaluronic acid-paclitaxel: effects of intraperitoneal administration against CD44(+) human ovarian cancer xenografts
}

\author{
Ilaria Stefano, Alessandra Battaglia, Gian Franco Zannoni, Maria Grazia \\ Prisco, Andrea Fattorossi, Daniele Travaglia, Silvia Baroni, Davide Renier, \\ Giovanni Scambia, Cristiano Ferlini, et al.
}

\section{To cite this version:}

Ilaria Stefano, Alessandra Battaglia, Gian Franco Zannoni, Maria Grazia Prisco, Andrea Fattorossi, et al.. Hyaluronic acid-paclitaxel: effects of intraperitoneal administration against CD44(+) human ovarian cancer xenografts. Cancer Chemotherapy and Pharmacology, 2010, 68 (1), pp.107-116. 10.1007/s00280-010-1462-2 . hal-00624427

\section{HAL Id: hal-00624427 \\ https://hal.science/hal-00624427}

Submitted on 17 Sep 2011

HAL is a multi-disciplinary open access archive for the deposit and dissemination of scientific research documents, whether they are published or not. The documents may come from teaching and research institutions in France or abroad, or from public or private research centers.
L'archive ouverte pluridisciplinaire HAL, est destinée au dépôt et à la diffusion de documents scientifiques de niveau recherche, publiés ou non, émanant des établissements d'enseignement et de recherche français ou étrangers, des laboratoires publics ou privés. 
HYALURONIC ACID-PACLITAXEL: EFFECTS OF INTRAPERITONEAL ADMINISTRATION AGAINST CD44(+) HUMAN OVARIAN CANCER XENOGRAFTS.

Ilaria De Stefano ${ }^{\mathrm{a}}$, Alessandra Battaglia $^{\mathrm{a}}$, Gian Franco Zannoni ${ }^{\mathrm{b}}$, Maria Grazia Prisco ${ }^{\mathrm{a}}$, Andrea Fattorossi ${ }^{\mathrm{a}}$, Daniele Travaglia ${ }^{\mathrm{a}}$, Silvia Baroni ${ }^{\mathrm{c}}$, Davide Renier ${ }^{\mathrm{d}}$, Giovanni Scambia ${ }^{\mathrm{a}}$, Cristiano Ferlini ${ }^{\mathrm{a}}$, and Daniela Gallo ${ }^{\mathrm{a}}$

${ }^{\mathrm{a} D}$ Department of Obstetrics and Gynaecology, ${ }^{\mathrm{b}}$ Department of Histopathology, ${ }^{\mathrm{c}}$ Institute of Clinical Biochemistry, Catholic University of the Sacred Heart, Largo A. Gemelli, 8, 00168 Roma, Italy. ${ }^{\mathrm{d}}$ Fidia Farmaceutici S.p.A. Via Ponte della Fabbrica 3/A, 35031 Abano Terme (PD) Padova, Italy.

\section{Correspondence to:}

Dr. Daniela Gallo

Department of Obstetrics and Gynaecology, Catholic University of the Sacred Heart, Largo A. Gemelli, 8 - 00168 - Rome - Italy

Tel +3906 3013337; FAX +39063051160

e-mail: d.gallo@rm.unicatt.it 


\section{ABSTRACT}

Purpose. Hyaluronan (HA)-receptors (mainly CD44 and RHAMM) are overexpressed in a wide variety of cancers including ovarian tumours, and HA-bioconjugates have been developed to enhance selective entry of cytotoxic drugs into HA-receptor expressing cancerous cells. Here we evaluated the potential application of a new HA-paclitaxel bioconjugate, ONCOFID-P, for intraperitoneal (IP) treatment of ovarian cancer. Methods. In vitro cytotoxic effect of ONCOFID-P, was first assessed on CD44(+) OVCAR-3 and SKOV3 human ovarian cancer cell lines. In vivo studies were performed in female Balb/c athymic mice IP implanted with OVCAR-3 or SKOV-3 and treated with IP ONCOFID-P, and IP and intravenous (IV) free paclitaxel, at their Maximum Tolerated Dose (MTD 168, 80 and 80 $\mathrm{mg} / \mathrm{kg}$, total dose, respectively). The potential detrimental effect of the IP ONCOFID-P and IP free paclitaxel on haematopoiesis was also assessed on peripheral blood, bone marrow and spleen. Results. Results show that ONCOFID-P cytotoxicity against both OVCAR-3 and SKOV-3 cell lines was somewhat less effective than free paclitaxel. Conversely, in in vivo experiments, IP treatment with ONCOFID-P was overall more effective than IV and IP free paclitaxel in inhibiting intra-abdominal tumour dissemination, abrogating ascites, prolonging survival and curing mice. ONCOFID-P and IP free paclitaxel were equivalent in terms of myelotoxicity, although the former was administered at a two-fold higher dose. Conclusions. Present data strongly support the development of ONCOFID-P for locoregional treatment of ovarian cancer.

Keywords: Hyaluronan, Paclitaxel, CD44, Mice, Ovarian cancer. 


\section{INTRODUCTION}

Epithelial ovarian cancer is the leading cause of death for gynaecological cancer in most of the Western world. It is the ninth most common cancer and the fifth leading cause of cancer death among women in the USA [1]; in European women it accounts for 41900 cases (about $3.9 \%$ of all female cancers) [2]. Ovarian cancer is recognized to be one of the most chemotherapy-sensitive malignancies, with $70-80 \%$ of newly diagnosed patients being anticipated to exhibit a response to primary platinum plus taxane chemotherapy; despite this fact, the majority of women who present with advanced ovarian cancer will experience recurrence, and ultimately die of complications associated with progressive disease [3]. The peritoneum is the predominant site of failure, and intraperitoneal spread of disease is present in patients with early recurrence. In this regard, the regional delivery of chemotherapeutic agents into the peritoneal cavity may provide a pharmacologic advantage, by directly exposing tumour to greater concentrations of anti-neoplastic drugs, and for a longer period, compared to systemic therapy [4]. It is still unknown, however, whether the antitumour effect is mainly related to direct drug tumour penetration, prolonged drug exposure, higher serum drug levels due to absorption, or if IP chemotherapy modifies the immune response against tumour cells in the peritoneal cavity [5]. Patients with microscopic or small volume residual disease seem to be most suitable for this approach, while, in more bulky disease, IP chemotherapy is unlikely to be beneficial because drug penetration is limited [6].

Cisplatin has been most frequently used for IP chemotherapy in ovarian cancer patients, with Phase-III-trial data revealing, in small-volume residual advanced ovarian cancer, a superior efficacy of this treatment modality compared with systemic platinum delivery. However, this approach has been associated with greater toxicity both owing to the use of cisplatin (rather than carboplatin), and the requirement for IP catheter placement for drug delivery [7]. Paclitaxel and docetaxel are also promising drugs for IP chemotherapy in ovarian cancer, for several reasons. They have a considerable cytotoxic activity against ovarian cancer, and show 
favourable pharmacokinetics after IP administration: in addition, since they have a dosedependent response, the high locoregional drug concentrations should theoretically lead to increased cytotoxicity. Recently, feasibility and efficacy of this treatment have been demonstrated in a number of clinical studies [8].

An additional strategy to improve antitumour efficacy of traditional treatments is based on the use of delivery systems, as liposomes or other carriers, which allow selective drug accumulation in tumour tissues, tumour cells, or even compartments of tumour cells. By increasing bioavailability of drugs at sites of action, this approach may even enhance efficacy against resistant tumours [9]. Hyaluronan (HA) is a linear polymer, composed of repeating disaccharides of glucuronic acid and $\mathrm{N}$-acetyl-d-glucosamine that can reach a molecular mass of several million Daltons [10]. So far, cell receptors that have been identified for HA fall into three main groups: CD44, RHAMM (Receptor for HA Mediated Motility) and ICAM-1 (Intracellular adhesion molecule-1) [10, 11]. HA receptors (CD44, RHAMM) are overexpressed in a wide variety of cancers including ovarian tumours [12-14]. Targeting of anti-cancer agents to tumour cells and tumour metastases can thus be accomplished by receptor-mediated uptake of bioconjugates of anticancer agents and HA, followed by the release of free drugs through the degradation of HA in cell compartments. HA-drug bioconjugates may present a markedly enhanced selectivity for cancerous cells, while exhibiting improved water solubility relative to the parent drug [15]. However, since CD44 is also expressed by haemopoietic stem/progenitor cells and on cells of the haemopoietic microenvironment [16-18], it is important to ascertain whether HA-bioconjugates exert a more toxic effect than their parent compounds on the haemopoietic tissue.

The present study extends previous findings on the development of a new HA-paclitaxel bioconjugate (ONCOFID-P) for IP treatment of ovarian cancer [19]. Our research was aimed at evaluating drug activity at the Maximum Tolerated Dose (MTD) identified in preclinical toxicological studies for regulatory submission $(210 \mathrm{mg} / \mathrm{kg} /$ day, Fidia Farmaceutici, internal 
data). We also verified the potential effect of the tested substance on peripheral blood, bone marrow cellularity and spleen morphology. Results obtained confirmed that ONCOFID-P has a significant therapeutic effectiveness, and it advantages respect to conventional paclitaxel therapy (standard intravenous treatment). We also showed that ONCOFID-P proved to be more effective than free IP paclitaxel, and exhibited similar side effects at a 2-fold higher dose. Importantly, the relatively high water-solubility of the bioconjugate eliminates the requirement of Cremophor EL as a solvent, with its recognized irritating and toxic properties [20]. 


\section{MATERIALS AND METHODS}

\section{Drugs}

The preparation of HA-paclitaxel bioconjugate (ONCOFID-P, Fidia Farmaceutici, Abano Terme, Italy) with $\sim 20 \%$ w/w of paclitaxel loading has been previously described [21]. Briefly, HA (Mw $\sim 200 \mathrm{kDa}$ ) was employed to produce the tetrabutylammonium-hyaluronic salt used for the reaction with the paclitaxel ester generated by activation with 4-bromo butyric acid. For in vitro studies ONCOFID-P ${ }^{\circledR}$ and paclitaxel (donated by Fidia Farmaceutici, Padova, Italy) were diluted in DMSO. Solutions were further diluted at each experimental day in order to achieve a $0.1 \%$ final DMSO concentration. All reagents were purchased from Sigma (Sigma- Chemical Co. St. Louis, Mo.), unless indicated.

\section{Cell Lines}

OVCAR-3 and SKOV-3 cells were purchased from the European Collection of Cell Cultures (ECACC, Salisbury, UK). OVCAR-3 were grown in RPMI 1640 medium supplemented with $10 \%$ foetal bovine serum, $1 \%$ non-essential amino acids mixture, $1 \%$ Kanamycin. SKOV-3 were grown in McCoy's medium supplemented with $10 \%$ foetal bovine serum, $1 \% \mathrm{MEM}, 1 \%$ Kanamycin. Cells, propagated as a monolayer culture, were trypsinised twice weekly and plated at a density of $10 \times 10^{4}$ cells per ml. All cultures were incubated at $37{ }^{\circ} \mathrm{C}$ under $5 \%$ $\mathrm{CO}_{2}$ in a high humidity atmosphere. On the day of tumour inoculation, cells were trypsinised, collected in RPMI 1640 medium without supplements and a suspension of $8 \times 10^{6}$ cells were injected intraperitoneally in each animal $(0.2 \mathrm{ml}$ per mice $)$.

\section{Growth inhibition assay}

Cells were seeded (20 000 cells/well) in 96-well flat bottom plates (Viewplates, Perkin Elmer Life Science, Waltham, MA). After 24 hours, media were replaced and, after one washing, media containing the test compounds were added. Three independent experiments were 
performed in quadruplicates. After $72 \mathrm{~h}$ of culture in the presence of the compounds, plates were harvested and the number of viable cells was estimated by dosing ATP, using the ATPlite kit (Perkin-Elmer Life Science), and the automated luminometer Topcount (PerkinElmer Life Science). For each compound a dose-response curve was plotted, and the IC50 values were then calculated by fitting the concentration-effect curve data obtained in the three experiments with the sigmoid-Emax model using nonlinear regression, weighted by the reciprocal of the square of the predicted effect [22].

\section{In vivo studies}

\section{$\underline{\text { Animals and treatments }}$}

Female athymic mice (Balb/c nu/nu 4-5 weeks old) were purchased from Charles River, S.r.l. (Calco, Lecco, Italy) and housed under controlled conditions. Procedures and facilities followed the requirements of Commission Directive 86/609/EEC concerning the protection of animals used for experimental and other scientific purposes. Italian legislation is defined in the Decreto Legislativo No. 116 of 27 January 1992. In addition the UKCCCR guidelines for the welfare of animals in experimental neoplasia were followed [23]. Studies were approved by the Animal Care and Use Committee of the Catholic University of the Sacred Heart (Rome, Italy), and by the Italian Ministry of Health.

\section{Efficacy studies (OVCAR-3 and SKOV-3)}

On the day of inoculation, a suspension of OVCAR-3 or SKOV-3 (8 x 10 $0^{6}$ cells) was injected intraperitoneally in athymic mice. ONCOFID-P was formulated daily by dissolution in glucosate $5 \%(\mathrm{w} / \mathrm{v}$ in physiological saline) at a concentration of $15 \mathrm{mg} / \mathrm{ml}$. A stock solution of paclitaxel (concentration of $20 \mathrm{mg} / \mathrm{ml}$ ) was prepared by dissolving it into equal volumes of absolute ethanol and Cremophor. The stock solution was prepared once per week and stored at $4^{\circ} \mathrm{C}$. Immediately before administration, the stock solution was further diluted with 
physiological saline to give the concentration required for dosing (i.e. $2 \mathrm{mg} / \mathrm{ml}$ ). ONCOFID-P and its vehicle were administered by the IP route at a dose volume of $14 \mathrm{ml} / \mathrm{kg}$; paclitaxel was administered either IV or IP at a dose volume of $10 \mathrm{ml} / \mathrm{kg}$. Dose volume was calculated on the basis of the most recently recorded body weight. For each experimental model, a total of 4 experimental groups were used: Control (glucosate 5\%, days 8, 15, 30, 37, IP), IP ONCOFID$\mathrm{P}(210 \mathrm{mg} / \mathrm{kg} /$ injection, days $8,15,30,37)$, IP free paclitaxel (20 mg/kg/injection, days 8,15 , $30,37)$ and IV free paclitaxel (20 mg/kg/injection, days $8,11,15,18)$. ONCOFID-P regimen was chosen according to preliminary experimental data (unpublished results). IV paclitaxel was administered at the MTD, and according to a conventional schedule treatment able to achieve a $60 \%$ cure rate in the sensitive A2780 ovarian cancer model [24]. IP paclitaxel was administered at its MTD determined in preliminary studies (unpublished results), with the same regimen as ONCOFID-P.

During the study, mice were checked daily for any adverse clinical reactions and body weights were measured twice a week. Each experimental group included 15 mice. In the OVCAR-3 study, 10 mice/group were used for evaluating survival, while the remaining 5 were sacrificed 6 weeks following tumour inoculation (day 42 of the study) to assess rate of tumour progression by measurement of tumour size at necropsy and possible evidence of ascites. As IP ovarian xenografts disseminate throughout the peritoneal cavity, all macroscopic (visible) nodules were excised harvested and weighed, followed by fixation in neutral buffered $10 \%$ formalin, and subsequent dehydratation and embedding in paraffin. The paraffin block was cut into 3 or $5 \mu \mathrm{m}$ sections, and processed for light microscopy (hematoxylin/eosin staining, H\&E) or immunohistochemistry for evaluation of CD44 expression (see below). Blood was collected from the caudal vena cava and subsequently used for assessment of CA 125 levels. Liver, kidneys and spleen were weighed for all mice. 


\section{CD44 Immunohistochemistry}

Three-micrometer-thick paraffin sections were mounted on Superfrost coated slides, and dried overnight. The sections were deparaffinized in xylene and rehydrated in graded solutions of ethanol; the endogenous peroxidase was blocked with $3 \% \mathrm{H}_{2} \mathrm{O}_{2}$ for 5 min. The antigen retrieval procedure was performed by microwave oven heating in $10 \mathrm{mM}$ citric acid, $\mathrm{pH} 6$ (3 times for $5 \mathrm{~min})$. To reduce non specific binding the sections were incubated with $20 \%$ normal goat serum for $30 \mathrm{~min}$, at room temperature. To detected expression of CD44, sections were stained overnight at $4^{\circ} \mathrm{C}$, with rabbit monoclonal CD44, clone EPR1013Y, (Abcam Inc., Cambrige, MA, UK) at 1:100 dilution; sections were incubated with the secondary, anti-rabbit EnVision System-HRP (DakoCytomation, Carpinteria, CA, USA) for $30 \mathrm{~min}$, at room temperature. The slides were developed with diaminobenzidine (DAB substrate System, DakoCytomation), counterstained with Mayer's Haematoxylin, dehydrated in ethanol and xylene, and finally mounted.

\section{$\underline{\text { CA } 125 \text { measurement }}$}

The serum levels of CA 125 antigen were measured by Access ${ }^{\circledR}$ OV MONITOR (Beckman Coulter) noncompetitive immunochemiluminescent assay, using the Beckman Coulter UniCel DxI 800 analyzer. The OV Monitor assay uses alternative antibodies (OVK95 and OV185) rather than the traditional OC125 and M11 antibodies for the detection of CA $125[25,26]$. The minimum detectable concentration (MDC) is calculated to be $0.1 \mathrm{U} / \mathrm{ml}$ [25]. All studied samples were assayed in duplicate; moreover, all mouse sera were measured undiluted and appropriately diluted for evaluating the possible presence of circulating protein interferants.

\section{Safety study}

In this study we compared the haematological toxicity of ONCOFID-P and IP free paclitaxel by assessing changes on peripheral blood, bone marrow and spleen. To this end, female 
athymic mice (Balb/c nu/nu, $\mathrm{n}=5$ per group) were allocated to 3 experimental groups: control untreated, IP ONCOFID-P (210 mg/kg/injection, days 8, 15, 30, 37), and IP free paclitaxel (20 mg/kg/injection, days 8, 15, 30, 37). Mice were sacrificed one week after the last dosing (day 42, see above). Blood was collected at necropsy from the caudal vena cava and bone marrow cells were isolated by flushing the femoral bones with PBS (phosphate buffered saline). Spleens were weighed and processed for light microscopy (hematoxylin/eosin staining, H\&E).

For peripheral blood assessment, at least 300ul of blood sample was drawn from caudal vena cava. Total leukocyte and differential count were performed. Briefly, leukocytes were incubated with buffered $\mathrm{NH}_{4} \mathrm{Cl}$ (blood/ $\mathrm{NH}_{4} \mathrm{Cl}$ ratio 1:20) at room temperature for 10 minutes. Leukocytes were then centrifuged at 350xg for $10 \mathrm{~min}$, washed once with phosphate buffered saline (PBS) and resuspended in $2 \mathrm{ml}$ of the same buffer. Granulocytes lymphocytes and monocytes were measured using the forward and side scatter signals in a Beckman-Coulter XL flow cytometer. Forward and side scatter signals were collected in linear mode and served also to exclude unwanted events (i.e., not viable cells, debris and cell clumps) from cell evaluation. A minimum of $5 \times 10^{3}$ up to $15 \times 10^{3}$ cells of interest were acquired for each sample. List mode data were analyzed using Expo 32 ${ }^{\mathrm{TM}}$ (Beckman Coulter) software. Total white blood cell (WBC) count per cubic millimeter was obtained using a Z2 Coulter Particle Count and Size analyzer (Beckman Coulter). Leukocyte subset proportions were converted to the absolute number per cubic millimeter multiplying each cell percentage by the number of leukocyte per cubic millimeter derived from the WBC count.

The marrow was passed through a 26-gauge needle to generate single cell suspensions and erythrocytes lysed using buffered $\mathrm{NH}_{4} \mathrm{Cl}$. $\mathrm{BM}$ cells were then counted using a $\mathrm{Z} 2$ Coulter Particle Count and Size analyzer. 


\section{$\underline{\text { Statistical methods }}$}

To determine significant differences in survival curves between groups, the Kaplan-Meier survival analysis was used, followed by logrank test. Body weight data were analyzed by the repeated-measures ANOVA, followed by the Bonferroni method as post-test. Tumour incidence in the satellite groups was compared using a Chi-square test. All the remaining data were analyzed for homogeneity of variance using Bartelett's test. If the group variance appeared homogenous, a parametric ANOVA was used, followed by Tukey's multiple comparison test. If the variances were heterogeneous, log or reciprocal transformations were made in an attempt to stabilize the variances. If the variances remained heterogeneous a non parametric test such as the Kruskall-Wallis test, followed by Dunn's multiple comparison test, was used. Statistical analysis was carried out with GraphPad Prism5 Software (San Diego, CA, USA). Values in the text are means \pm SEM. P $<0.05$ was used as the critical level of significance. 


\section{RESULTS}

\section{In vitro tumour growth inhibition}

ONCOFID-P was tested against OVCAR-3 and SKOV-3 ovarian cancer cell lines, and its activity was compared with that of free paclitaxel. ONCOFID-P yielded IC50 values of $642 \pm$ 284, and $820 \pm 330 \mathrm{ng} / \mathrm{ml}$, following 72 hours drug exposure of OVCAR-3 and SKOV-3, respectively (corresponding to $128 \pm 57$ and $164 \pm 66 \mathrm{ng} / \mathrm{ml}$ in paclitaxel equivalent). Unconjugated paclitaxel produced IC50 values of $41.1 \pm 24.4$ and $2.8 \pm 0.7 \mathrm{ng} / \mathrm{ml}$, for OVCAR-3 and SKOV-3, respectively. Collectively results showed that the bioconjugate exhibited obvious cytotoxicity against both OVCAR-3 and SKOV-3 ovarian cancer lines, i.e., paclitaxel is released from the tested conjugate without losing cytotoxicity, although its antitumour activity in vitro was lower than that of pure paclitaxel.

\section{In vivo anti-tumour efficacy - OVCAR-3 study}

Treatment with $210 \mathrm{mg} / \mathrm{kg} /$ injection ONCOFID-P (corresponding to $42 \mathrm{mg} / \mathrm{kg} /$ injection in paclitaxel equivalent) had a significant therapeutic activity, resulting in a 2.5 -fold increase in survival in comparison to control mice: specifically, median survival was 113.5 vs 45.5 days in controls $(\mathrm{p}<0.001)$ (Fig. 1A). IP treatment with free paclitaxel $(20 \mathrm{mg} / \mathrm{kg} /$ injection $)$ also produced a significant therapeutic benefit (median survival, 89 days, $\mathrm{p}<0.001$ vs controls), with a $2.0-$ fold increase in survival over control. Notably, on day 189 of the study (i.e., 5 months after the end of treatment), there were two tumour free cures in the ONCOFID-P group, and one in the IP free paclitaxel group. On the other hand, the conventional IV paclitaxel $(20 \mathrm{mg} / \mathrm{kg} /$ injection $)$ did not significantly increase survival in comparison to controls (median survival 67.5 days, $\mathrm{p}=0.23$ ). Body weight curves during the study are reported in Figure 1B. Administration of ONCOFID-P did not significantly affect body weight of mice throughout the study period. Conversely, animals receiving IP free paclitaxel lost weight upon treatment, this change achieving statistical significance on weeks 2 and 3 
$(\mathrm{p}<0.05)$. Similarly, paclitaxel IV-treated mice had a group mean body weight significantly lower than control $(\mathrm{p}<0.05)$ from week 2 until week 7 (end of treatment-period, and last statistical analysis, surviving control mice $=5$ ).

\section{$\underline{\text { Interim sacrifice- Satellite groups }}$}

Figure 1C shows representative pictures of mice in the different treatment groups 6 weeks after tumour inoculation and Figure 1D shows histological features and CD44 expression of OVCAR-3 tumours collected at the interim sacrifice. At this time, tumour was evident in all mice from control, IP free paclitaxel and IV free paclitaxel (100\%), while only 2 out of 5 ONCOFID-P-treated mice (40\%) showed evidence of tumour $(\mathrm{p}<0.05$ vs control, Fig. 1E). Mean tumour weight in ONCOFID-P and IP paclitaxel group were significantly lower than control ( $\mathrm{p}<0.05)$; a trend towards a reduction in tumour burden was also observed in the IV free paclitaxel group, although this change did not reach statistical significance (Fig. 1F).

The presence of ascitic fluid was found in all mice in the control group (100\%), with a mean volume per mouse of $1.5 \pm 0.6 \mathrm{ml}$; conversely, there was no evidence of ascites either in ONCOFID-P- or IP free paclitaxel-treated mice $(\mathrm{p}<0.05)$. In the IV free paclitaxel group only one mouse showed ascites at necropsy (Fig. 1G).

The serum CA 125 level of tumour-bearing control mice was $145.9 \pm 90.9$ units $/ \mathrm{ml}$. By comparison, it was extremely lower in all treated groups, with mean levels of $0.2 \pm 0.2,0.0 \pm$ 0.0 , and $0.9 \pm 0.9$, for ONCOFID-P, IV free paclitaxel and IP free paclitaxel, respectively (Fig. 1H). The differences observed in CA 125 levels among treatment groups did not closely mirror changes measured in tumor burden, as a likely consequence of a low stage of the disease, at which the peritoneal barrier is efficient in controlling the release of tumor antigens in the circulation [27]. According to this, although elevated CA 125 levels occur in about $80 \%$ of patients with advanced ovarian cancer, the frequency of positivity is quite lower in low stage disease [28]. 
Liver, spleen and kidneys from all animals were weighed at necropsy. Results obtained showed no effect of the different treatments on both absolute and relative liver and kidney weights. Conversely, there was a significant increase in absolute and relative spleen weight of animals receiving ONCOFID-P and IP free paclitaxel when compared to controls $(\mathrm{p}<0.05$, Fig 1I and L).

\section{In vivo anti-tumour efficacy- SKOV-3 study}

The therapeutic activity of ONCOFID-P was confirmed using SKOV-3, although the effects observed were overall reduced, as a likely consequence of a fast tumour-growth rate. Specifically, treatment with $210 \mathrm{mg} / \mathrm{kg} /$ injection ONCOFID-P (corresponding to 42 $\mathrm{mg} / \mathrm{kg} /$ injection in paclitaxel equivalent) had a significant therapeutic activity, resulting in a

increase in survival in comparison to control mice, with a median survival of 50 versus 35 days in controls $(\mathrm{p}<0.001)$ (Fig. 2A). IP treatment with free paclitaxel $(20 \mathrm{mg} / \mathrm{kg} /$ injection $)$ also produced a slight increase in survival over control, although to a lesser extent (median survival, 39 days, $\mathrm{p}<0.05$ vs controls). Notably, differences between ONCOFID-P and IP free paclitaxel approached statistical significance $(p=0.057)$ IV free paclitaxel (20 $\mathrm{mg} / \mathrm{kg} /$ injection) did not affect survival (median survival 31 days, $\mathrm{p}=0.8$ ). On day 189 of the study (i.e. 5 months after the end of treatment), there were two tumour free cures in ONCOFID-P group. Body weight curves during the study are reported in Figure $2 \mathrm{~B}$. Administration of either ONCOFID-P and IP free paclitaxel did not significantly affect body weight of treated mice throughout the study period. Conversely, IV paclitaxel-treated mice had group mean body weight significantly lower than controls on weeks 3 and $4(\mathrm{p}<0.05)$. Figure 2C shows histological features and CD44 expression of SKOV-3 tumours. 


\section{Safety evaluations}

WBC count in control mice averaged around $2.83 \pm 0.37 \times 10^{6}$ cells $/ \mathrm{ml}$. Both treatments significantly reduced WBC counts to a similar extent ( $\mathrm{p}<0.01$, Fig. 3A). This effect was accounted for by a significant decrease in both lymphocyte and polymorphonuclear cell counts, whereas monocyte count was not modified by either treatment (Fig. 3B). ONCOFIDP and free paclitaxel by the same route of dosing did not affect BM cellularity (Fig. 3C).

When compared with controls, mice receiving IP free paclitaxel showed a significant increase in both absolute and relative spleen weights ( $\mathrm{p}<0.001$ for both parameters), while ONCOFIDP- treated mice only showed a significant increase in the absolute organ weight $(\mathrm{p}<0.05)$ (Fig. 3D). Noteworthy, both absolute and relative spleen weights of IP free paclitaxel-treated mice were significantly higher than those measured in ONCOFID-P-treated mice $(\mathrm{p}<0.01)$. Histological evaluation showed that when compared with controls, treated mice showed a modest but consistent red pulp expansion, with a more prominent extramedullary haematopoiesis, which is typical of mice spleen [29]. Changes induced by ONCOFID-P (Fig. 3E) and IP free paclitaxel (not shown) were comparable. 


\section{DISCUSSION}

Results from the present study are in line with and extend data of previous reports demonstrating that ONCOFID-P affords a potent in vivo therapeutic activity when tested in preclinical models of ovarian cancer [19]. We purposely evaluated bioconjugate activity in two different in vivo experimental models closely reflecting disease progression in human ovarian cancer, with tumour dissemination throughout the peritoneum and ascites production [30]. Results obtained showed that, in both experimental models, IP treatment with ONCOFID-P at the MTD inhibited intra-abdominal tumour dissemination and production of ascites, resulting in prolonged survival and cured mice. Conversely, therapy at the MTD with free paclitaxel by the IV route, which is still the standard administration route of the drug, was completely devoid of efficacy, while exerting toxicity, as demonstrated by the body weight loss observed. Notably, administration of free paclitaxel by the IP route although increased effectiveness in comparison to free drug given by the IV route, was however still less efficient than ONCOFID-P administration, in both experimental models used. These in vivo findings are seemingly not in line with our in vitro data showing that, in our experimental conditions, antiproliferative activity of ONCOFID-P was lower than that of pure paclitaxel. This, however, is not a surprising result, as the advantages of ONCOFID-P over paclitaxel are likely to be more prominent in vivo than in vitro. Among the valuable factors, a significant role is certainly played by the favourable pharmacokinetic behaviour of the bioconjugate compared to that of the free drug, with different critical outcomes in terms of distribution pattern. Indeed, pharmacokinetic studies indicated that while following IP free drug administration, the plasma concentrations peaked in $6 \mathrm{hrs}$ and were almost negligible at 24 hrs, after IP ONCOFID-P there was a striking increase in drug plasma levels which plateaued within $24 \mathrm{hrs}$, lasted up to $48 \mathrm{hrs}$, and declined slowly in the following days, returning to basal levels only at $120 \mathrm{hrs}$ [19]. Thus, it is very likely that the IP injected bioconjugate remains in the peritoneal cavity allowing a direct, selective and long-lasting (several days) 
interaction with ovarian cancer cells widely expressing the CD44 receptor [14, 31], so maximizing drug uptake and therapeutic effectiveness. Further studies on tumor drug accumulation are needed to definitively confirm this advantage. It is worthy to note that previous investigations have already demonstrated an important pharmacokinetic advantage for free paclitaxel after IP regional delivery, when compared to the standard IV route [8], and other studies showed that conjugation with hyaluronic acid further extend the retention time of paclitaxel to the abdominal cavity [32]. However, for free IP paclitaxel effective dosing is impaired by the local dose-limiting toxicity [8]. In this respect, the relatively high watersolubility of the bioconjugate, eliminating the requirement of Cremophor EL as a solvent, with its recognized irritating and toxic properties [20], allows the administration of higher dosages. Collectively, the increased injection dose, the longer duration, and the enhanced local retention of the bioconjugate give support to the higher in vivo efficacy showed by ONCOFID-P even compared with IP paclitaxel.

It is worth mentioning that one of the advantages of ONCOFID-P over free paclitaxel resides in its enhanced capability to enter tumour cells via cell surface CD44. Paradoxically, the CD44 binding capability of ONCOFID-P might entail a modification of the side-effect profile as compared to free paclitaxel, due to the expression of CD44 also on the cell surface of leukocytes and haematopoietic stem/progenitor cells. This possibility seems remote however as ONCOFID-P, although administered at a 2-fold higher dose, proved not to be more myelotoxic than free IP paclitaxel. Interestingly enough, we observed that while at the time of sacrifice bone marrow cellularity had already recovered in both treatment groups, a modest expansion of the spleen red pulp was still present in treated mice, likely reflecting the occurrence of an enhanced and long-lasting extra medullary haematopoiesis attempting to control the myelotoxic effect induced by both treatments. This observation is well in line with the notion that in mice red pulp is physiologically involved in haematopoiesis [29], and overall, our data are in keeping with previous results showing that the bone marrow and the 
spleen display different time-courses of functional recovery in murine haematopoiesis post paclitaxel injury $[33,34]$. On the whole, changes observed imply that ONCOFID-P does not perform significantly worse than its comparator in terms of haematological toxicity. In this context, it is worth pointing out that myelosuppression is the dose-limiting toxicity for most chemotherapeutic drugs, including paclitaxel, and infection remains a common cause of death in chemotherapy treated patients. Thus, besides improvement in efficacy, the attainment of a reduction in toxicity represents a goal in the development of new taxanes or taxane analogues. Notably, macromolecular drug delivery systems have been developed as one approach to improve the therapeutic index and possibly overcome drug resistance.

In conclusion, results from our study, in keeping with previous literature data, strongly support the development of ONCOFID-P for locoregional treatment of ovarian cancer. This novel paclitaxel formulation shows to be a more tolerable, and safer method for drug administration, capable of providing higher dosages without increasing adverse effects. Furthermore, this delivery system demonstrates greater therapeutic efficacy in two different experimental models of human ovarian cancer. Importantly, a phase I study is now ongoing in our Institution to investigate the MTD and the safety profile of ONCOFID-P following IP infusion in patients affected by intraperitoneal carcinosis due to ovarian, breast, stomach, bladder and colon cancer. Further studies should be also envisaged to investigate the feasibility of using a metronomic rather than a MTD schedule for this drug. 


\section{Conflict of interest statement}

This work has been supported by a grant from Fidia Farmaceutici, Abano Terme, Italy. The funding sources had no role in the study design, data collection, data analysis, data interpretation, writing of this report or in the decision to submit the paper for publication. 


\section{REFERENCES}

1. Jemal A, Siegel R, Ward E, Hao Y, Xu J, Thun MJ (2009) Cancer statistics. CA Cancer J Clin 59:225-49.

2. Ferlay J, Autier P, Boniol M, Heanue M, Colombet M, Boyle P (2007) Estimates of the cancer incidence and mortality in Europe in 2006. Ann Oncol 18:581-92.

3. Markman M (2008a) The promise and perils of 'targeted therapy' of advanced ovarian cancer. Oncology 74:1-6.

4. Landrum LM, Gold MA, Moore KN, Myers TK, McMeekin DS, Walker JL (2008) Intraperitoneal chemotherapy for patients with advanced epithelial ovarian cancer: a review of complications and completion rates. Gynecol Oncol 108:342-7.

5. Petignat P, du Bois A, Bruchim I, Fink D, Provencher DM (2007) Should intraperitoneal chemotherapy be considered as standard first-line treatment in advanced stage ovarian cancer? Crit Rev Oncol Hematol 62:137-47. Review.

6. de Bree E, Theodoropoulos PA, Rosing H, Michalakis J, Romanos J, Beijnen JH, Tsiftsis DD (2006a) Treatment of ovarian cancer using intraperitoneal chemotherapy with taxanes: From laboratory bench to bedside. Cancer Treat Rev 32:471-482.

7. Markman M (2008b) Antineoplastic agents in the management of ovarian cancer: current status and emerging therapeutic strategies. Trends Pharmacol Sci 29:515-9. Review.

8. de Bree E, Rosing H, Michalakis J, Romanos J, Relakis K, Theodoropoulos PA, Beijnen JH, Georgoulias V, Tsiftsis DD (2006b) Intraperitoneal chemotherapy with taxanes for ovarian cancer with peritoneal dissemination. Eur J Surg Oncol 32:666-70.

9. Mamot C, Drummond DC, Hong K, Kirpotin DB, Park JW (2003) Liposome-based approaches to overcome anticancer drug resistance. Drug Resist Updat 6:271-9. Review.

10. Lesley J, Hascall VC, Tammi M, Hyman R (2000) Hyaluronan binding by cell surface CD44. J Biol Chem 275:26967-75. 
11. Entwistle J, Hall CL, Turley EA (1996) HA receptors: regulators of signalling to the cytoskeleton. J Cell Biochem 61:569-77.

12. Kayastha S, Freedman AN, Piver MS, Mukkamalla J, Romero-Guittierez M, Werness BA (1999) Expression of the hyaluronan receptor, CD44S, in epithelial ovarian cancer is an independent predictor of survival. Clin Cancer Res 5:1073-1076.

13. Maxwell CA, McCarthy J, Turley E (2008) Cell-surface and mitotic-spindle RHAMM: moonlighting or dual oncogenic functions? J Cell Sci 121:925-32.

14. Chen H, Hao J, Wang L, Li Y (2009) Coexpression of invasive markers (uPA, CD44) and multiple drug-resistance proteins (MDR1, MRP2) is correlated with epithelial ovarian cancer progression. Br J Cancer 101:432-440.

15. Leonelli F, La Bella A, Migneco LM, Bettolo RM (2008) Design, synthesis and applications of hyaluronic acid-paclitaxel bioconjugates. Molecules 13:360-78.

16. Wilson JG (1997) Adhesive interactions in hemopoiesis. Acta Haematol 97:6-12. Review

17. Ghaffari S, Smadja-Joffe F, Oostendorp R, Lévesque JP, Dougherty G, Eaves A, Eaves C (1999) CD44 isoforms in normal and leukemic hematopoiesis. Exp Hematol 27:978-93.

18. Chan JY, Watt SM (2001) Adhesion receptors on haematopoietic progenitor cells. Br J Haematol 112:541-57.

19. Banzato A, Bobisse S, Rondina M, Renier D, Bettella F, Esposito G, Quintieri L, Meléndez-Alafort L, Mazzi U, Zanovello P, Rosato A (2008) A paclitaxel-hyaluronan bioconjugate targeting ovarian cancer affords a potent in vivo therapeutic activity. Clin Cancer Res 14:3598-606.

20. Gelderblom H, Verweij J, Nooter K, Sparreboom A (2001) Cremophor EL: the drawbacks and advantages of vehicle selection for drug formulation. Eur J Cancer 37: 1590-8.

21. Rosato A, Banzato A, De Luca G, Renier D, Bettella F, Pagano C, Esposito G, Zanovello P, Bassi P (2006) HYTAD1-p20: a new paclitaxel-hyaluronic acid hydrosoluble bioconjugate for treatment of superficial bladder cancer. Urol Oncol 24:207-15. 
22. Motulsky HJ, Ransnas LA (1987) Fitting curves to data using nonlinear regression: a practical and nonmathematical review. FASEB J 1:365-374.

23. United Kingdom Co-ordinating Committee on Cancer Research (UKCCCR) (1998) Guidelines for the Welfare of Animals in Experimental Neoplasia (Second Edition). Br J Cancer 77:1-10.

24. Ferlini C, Cicchillitti L, Raspaglio G, Bartollino S, Cimitan S, Bertucci C, Mozzetti S, Gallo D, Persico M, Fattorusso C, Campiani G, Scambia G (2009) Paclitaxel directly binds to Bcl-2 and functionally mimics activity of Nur77. Cancer Res 69:6906-14.

25. Gandon G, Quillien V, Godey F (2004) Evaluation of the new Access OV Monitor assay for the quantitative determination of CA 125 antigen levels. Immuno-analyse and Biologie specialisée 19:228-234.

26. Holdenrieder S, Molina R, Gion M, Gressner A, Troalen F, Auge JM, Zancan M, WycisloM, Stieber P (2008) Alternative antibody for the detection of CA 125 antigen: a European multicenter study for the evaluation of the analytical and clinical performance of the Access OV Monitor assay on the UniCel DxI 800 Immunoassay System. Clin Chem Lab Med 46:588-99.

27. Fleuren GJ, Nap M, Aalders JG, Trimbos JB, de Bruijn HW (1987) Explanation of the limited correlation between tumor CA 125 content and serum CA 125 antigen levels in patients with ovarian tumors. Cancer 60:2437-42.

28. Duffy MJ, Bonfrer JM, Kulpa J, Rustin GJ, Soletormos G, Torre GC, Tuxen MK, Zwirner M. (2005) CA125 in ovarian cancer: European Group on Tumor Markers guidelines for clinical use. Int J Gynecol Cancer 15:679-91.

29. Cesta MF (2006) Normal structure, function, and histology of mucosa-associated lymphoid tissue. Toxicol Pathol 34: 599-608. Review.

30. Garson K, ShawTJ, Clark KV, Yao DS, Vanderhyden BC (2005) Models of ovarian cancer-are we there yet? Mol Cell Endocrinol 239:5-26. 
31. Makrydimas G, Zagorianakou N, Zagorianakou P, Agnantis NJ (2003) CD44 family and gynaecological cancer. In Vivo 17:633-40.

32. Yamada J, Kitayama J, Tsuno NH, Yamashita H, Miyato H, Soma D, Otani K, Kamei T, Ishigami H, Hidemura A, Kaisaki S, Takahashi K, Nagawa H (2008) Intra-peritoneal administration of paclitaxel with non-animal stabilized hyaluronic acid as a vehicle-a new strategy against peritoneal dissemination of gastric cancer. Cancer Lett 272:307-15.

33. Juaristi JA, Aguirre MV, Carmuega RJ, Romero-Benítez M, Alvarez MA, Brandan NC (2001) Hematotoxicity induced by paclitaxel: in vitro and in vivo assays during normal murine hematopoietic recovery. Methods Find Exp Clin Pharmacol 23:161-7.

34. Aguirre MV, Todaro JS, Juaristi JA, Brandan NC (2010) Murine erythropoietic impairment induced by paclitaxel: Interactions of GATA-1 and erythroid Krüppel-like transcription factors, apoptotic related proteins and erythropoietin receptor. Eur $\mathbf{J}$ Pharmacol 636:42-51. 


\section{FIGURE LEGENDS}

Figure 1 - Effect of ONCOFID-P on tumour growth, dissemination, and ascites formation. Female athymic mice were inoculated IP with OVCAR-3 cells. One week after inoculation, the mice were randomized into four treatment groups: Controls (IP glucosate 5\%, days 8, 15, 30, 37), IP ONCOFID-P (210 mg/kg/injection, days $8,15,30,37)$, IP free paclitaxel (20 $\mathrm{mg} / \mathrm{kg} /$ injection, days $8,15,30,37)$ and IV free paclitaxel $(20 \mathrm{mg} / \mathrm{kg} /$ injection, days $8,11,15$, 18). Six weeks after tumour inoculation, five mice from each treatment group were sacrificed to evaluate tumour progression. A-B) Tumour growth and body weight curves in the main experimental groups. Values are means \pm SEM, $\mathrm{n}=10 . * \mathrm{p}<0.05$, and $* * * \mathrm{p}<0.001$ vs Controls. C) Representative images of mice in the different treatment groups, 6 weeks after tumour inoculation. D) Histological features and CD44 expression of OVCAR-3 tumours (Magnification 10X). E-H) Percent of mice with tumour, tumour burden, ascite volume and CA 125 serum levels, in the different treatment groups, at the 6-weeks interim sacrifice. The box extend to the $25^{\text {th }}$ and $75^{\text {th }}$ percentiles; the horizontal bars indicate median values, and whiskers minimum and maximum values $(\mathrm{n}=5)$. I-L) Absolute and relative organ weight in the same animals. Columns represent mean \pm SEM, $n=5$. ${ }^{*} \mathrm{p}<0.05$, and $* * \mathrm{p}<0.01$ vs Controls.

Figure 2 - Effect of ONCOFID-P on tumour growth in female athymic mice inoculated IP with SKOV-3 cells. One week after inoculation, the mice were randomized into four treatment groups: Controls (IP glucosate 5\%, days 8, 15, 30, 37), IP ONCOFID-P (210 $\mathrm{mg} / \mathrm{kg} /$ injection, days $8,15,30,37)$, IP free paclitaxel (20 mg/kg/injection, days $8,15,30,37)$ and IV free paclitaxel $(20 \mathrm{mg} / \mathrm{kg} /$ injection, days $8,11,15,18)$. A-B) Tumour growth and body weight curves in experimental groups. Values are means $\pm S E M, n=15$. $* \mathrm{p}<0.05$, and *** $\mathrm{p}<0.0001$ vs Controls. C) Histological features and CD44 expression of SKOV-3 tumours (Magnification 10X). 
Figure 3 - Effect of ONCOFID-P on haematopoiesis in female athymic mice. Mice were randomized into three treatment groups: Controls (IP glucosate 5\%, days 8, 15, 30, 37), IP ONCOFID-P (210 mg/kg/injection, days 8, 15, 30, 37), IP free paclitaxel (20 mg/kg/injection, days $8,15,30,37)$. A-B) WBC count and differential and $\mathbf{C}$ ) bone marrow cellularity in the different treatment groups. D) Absolute and relative spleen weight in the different treatment groups. Values are means $\pm \mathrm{SEM}, \mathrm{n}=5 . * \mathrm{p}<0.05, * * * \mathrm{p}<0.0001$ vs Controls, and a $\mathrm{p}<0.01 v s$ ONCOFID-P. E) White and red pulp distribution in the spleen of Controls (upper panel) and ONCOFID-P treated mice (lower panel). (Magnification 10X). IP free paclitaxel induced architectural changes (not shown) comparable to those induced by ONCOFID-P. 
Figure 1 - OVCAR-3 experiment

Main groups
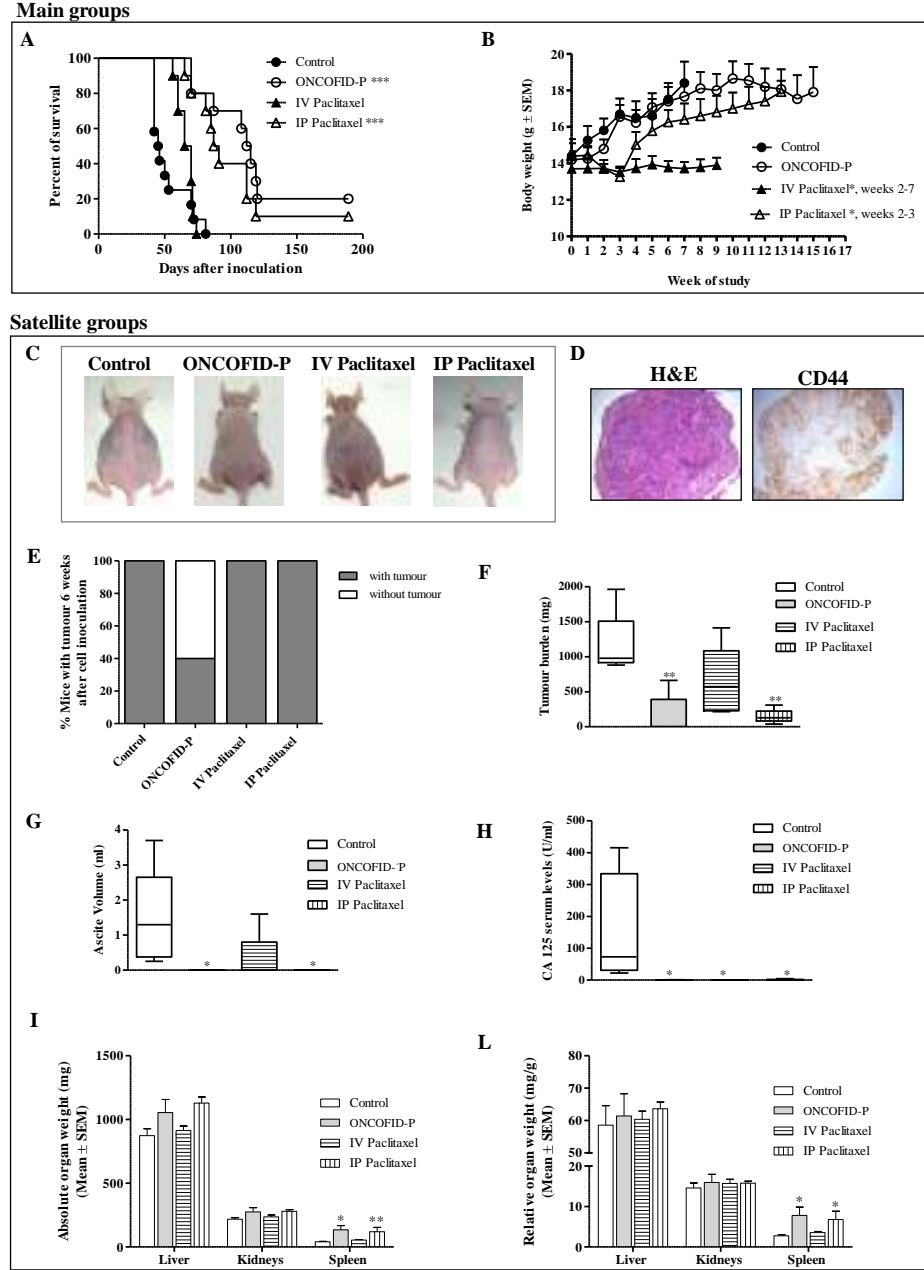
Figure 2 - SKOV-3 experiment

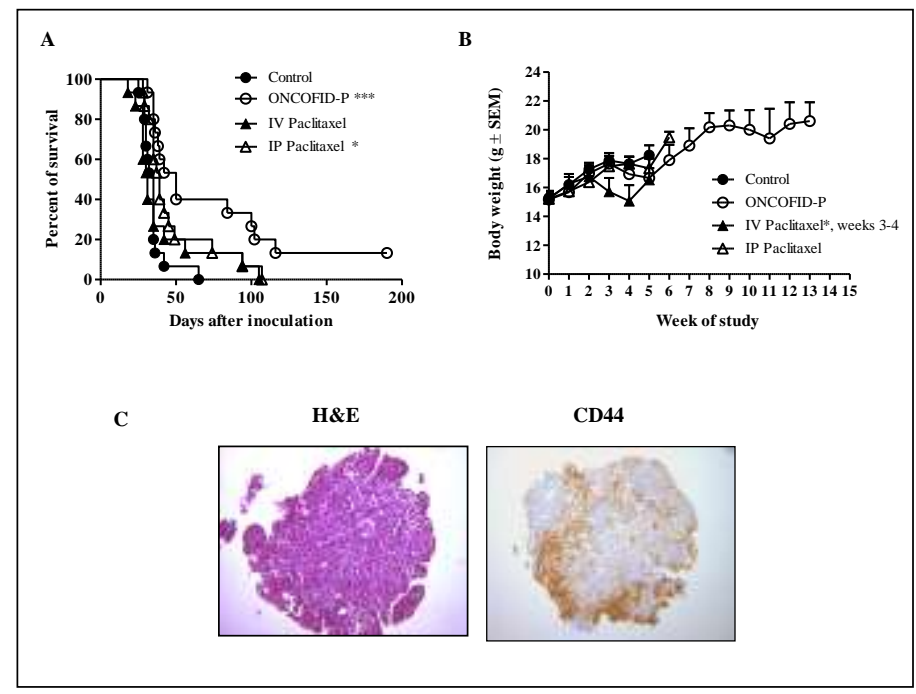


Figure 3 - Parameters of toxicity

Peripheral WBC Count and differential and Bone Marrow Cellularity

A

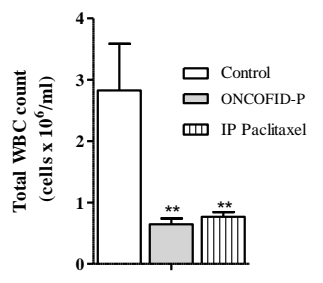

C
B
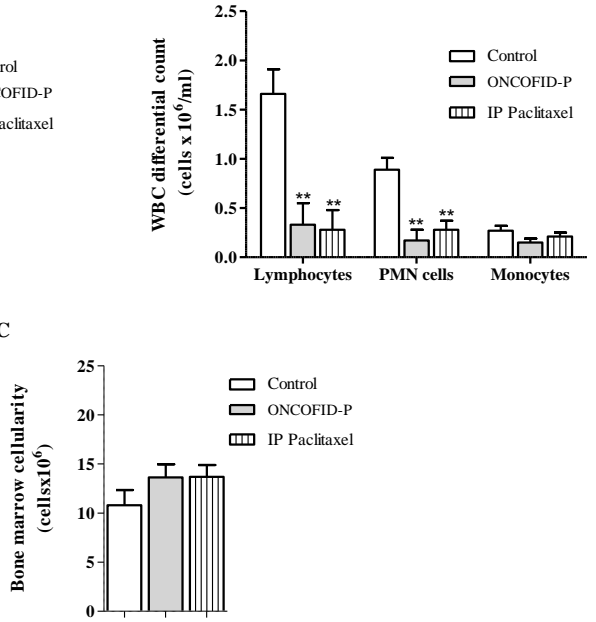

Spleen evaluation

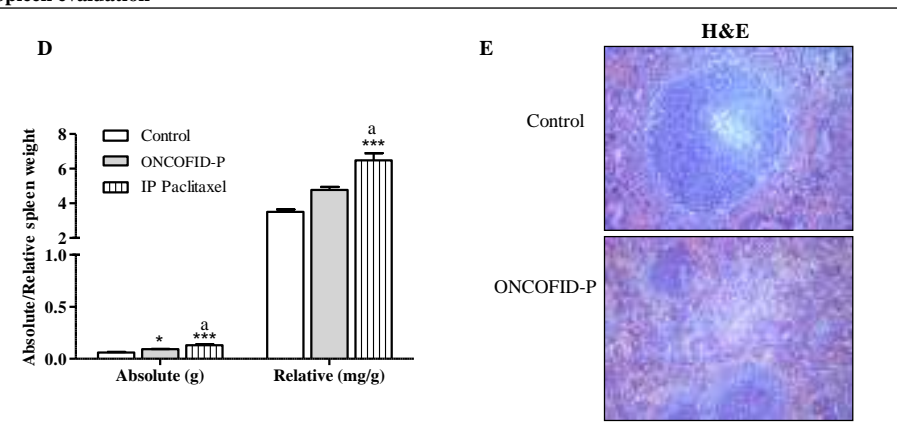

